\title{
Droplet deposition and spatial drift distribution characteristics of aerial spraying based on the determination of effective swath
}

\author{
Weixiang Yao ${ }^{1,2}$, Shuang $\mathrm{Guo}^{1,2}$, Fenghua $\mathrm{Yu}^{1,2}$, Wen $\mathrm{Du}^{1,2}$, Yanhua Meng ${ }^{3}$, Juan Wang ${ }^{4}$, \\ Pengchao Chen ${ }^{5}$, Xiang Li ${ }^{6}$, Tongyu $\mathrm{Xu}^{1,2^{*}}$, Yubin Lan ${ }^{5 *}$ \\ (1. College of Information and Electrical Engineering, Shenyang Agricultural University, Shenyang 110866, China; \\ 2. Liaoning Agricultural Information Engineering Technology Research Center, Shenyang 110866, China; \\ 3. College of Mechanical Engineering, Anyang Institute of Technology, Anyang, 455000, China; \\ 4. Mechanical and Electrical Engineering College, Hainan University, Haikou 570228, China; \\ 5. College of Electronic Engineering, College of Artificial Intelligence, South China Agricultural University, Guangzhou 510642, China; \\ 6. Jiangxi Tianren Ecology Co., Ltd, Jian 343100, China)
}

\begin{abstract}
In order to accurately explore the droplets distribution characteristics on the ground and space during the aerial spray process, a single-pass application test based on the determination of effective swath by a Bell206L4 helicopter was carried out. The accurate effective swath width of the helicopter spray operation was obtained, and the cumulative and spatial drift characteristics of droplet deposition when the helicopter used different aerial nozzles under natural environment were systematically analyzed. The results showed that the average effective swath width of three different types of nozzle were $25.3 \mathrm{~m}$ (CP02), $29.7 \mathrm{~m}$ (CP03) and $29.3 \mathrm{~m}$ (CP04) with an average flight altitude of $11 \mathrm{~m}$ and velocity of $17 \mathrm{~m} / \mathrm{s}$. The average deposition volume in the effective swath area of each sampling line was in the range of $1.208-2.735 \mu \mathrm{L} / \mathrm{cm}^{2}$. Most of the droplets in each application were concentrated near the route and the adjacent locations of the effective swath area. In addition, the droplets with small size were trend to drift, and the increased nozzle orifice size was beneficial to reduce drift. In terms of spatial drift distribution, small-sized droplets usually gathered in the upper space, larger-sized droplets were easy to gather in the middle space, and the medium-sized droplets were trend to gather in the lower space. The research results were helpful to optimize the spray plan and provide reference for precision aerial application.
\end{abstract}

Keywords: aerial spray, droplet deposition, spatial drift, effective swath, helicopter, precision agricultural DOI: $10.33440 /$ j.ijpaa.20210401.145

Citation: Yao W X, Guo S, Yu F H, Du W, Meng Y H, Wang J, Chen P C, et al. Droplet deposition and spatial drift distribution characteristics of aerial spraying based on the determination of effective swath. Int J Precis Agric Aviat, 2021; 4(1): $36-43$.

\section{Introduction}

The ensure and increase of crop yields is vital for social stability and economic development, and aerial pesticide application is a gradually emerged plant protection method that can ensure crop yields in recent years ${ }^{[1,2]}$. Aerial spraying involves

Received date: 2021-02-05 Accepted date: 2021-03-25

Biographies: Weixiang Yao, $\mathrm{PhD}$, research interests: precision agricultural aviation technology and equipment, Email: yaoweixiang@syau.edu.cn; Shuang Guo, Doctoral student, research interests: agricultural aviation technology, Email: 2358681011@qq.com; Fenghua Yu, PhD, research interests: precision agricultural aviation, Email: adan@syau.edu.cn; Wen Du, PhD, research interests: precision agricultural aviation, Email: duwen374@163.com; Yanhua Meng, PhD, research interest: precision agricultural aviation application, Email: 20200040@ayit.edu.cn; Juan Wang, PhD, Associate Professor, research interests: precision agricultural aviation, Email: 49792740@qq.com; Pengchao Chen, Doctoral student, Technician, research interests: precision agricultural technology and equipment, Email: 719777582@qq.com; Xiang Li, Agronomist, research interests: plant protection, Email: 550501889@qq.com.

*Corresponding author: Tongyu $\mathrm{Xu}, \mathrm{PhD}$, Professor, research interests: Precision agricultural aviation, Mailing Address: College of Information and Electrical Engineering, Shenyang Agricultural University, Shenyang, China. Email: xutongyu@syau.edu.cn.; Yubin Lan, PhD, Distinguished professor, Director, research interests: precision agricultural aviation application, Mailing Address: College of Electronic Engineering, College of Artificial Intelligence, South China Agricultural University, Guangzhou, China. Email: ylan@ scau.edu.cn. the dispersal of pesticides from high altitudes to the surface of the crop with the aid of a medium such as water or oil. The droplets are expected to be deposited at the location of pests and diseases and reduce drift $^{[3-5]}$.

Affected by aerial spraying conditions and airflow, pesticide drift phenomenon is easily occurred, which result in an effective droplet deposition weakened ${ }^{[6,7]}$. Many scholars have conducted researches on it. Thomson et al. ${ }^{[8]}$ used water sensitive paper evaluated the droplet deposition of an airplane equipped with a flow control system when spraying from different directions, and found that the direction of travel had a non-significant effect on the magnitude of spray deposition position error. Fritz ${ }^{[9]}$ documented the effects of atmospheric conditions and stability on the deposition and drift of aerially applied sprays. Yao et al. ${ }^{[10,11]}$ studied the atomization characteristics of LICHENG and $\mathrm{CP}$ aerial nozzles equipped by helicopters in the wind tunnel, and found that airflow velocity and spray pressure had a significant impact on the droplet size and deposition. There are also related studies on the droplet deposition effect under different application target. Meng et al. ${ }^{[12]}$ took peach trees as the application target, studied the aerial spray effects of different operating parameters and compared the sedimentation differences of two tree shapes. Wang et al. ${ }^{[13]}$ conducted experiments on the droplet deposition and drift characteristics of UAV spraying areca, and found that the flying height had a significant influence on the droplets, and the farthest 
droplet drift distance can reach 17.4 meters. Tang et al. ${ }^{[14]}$ studied the influence of tree shape and flight height on the deposition effect of UAV spraying citrus trees. The results showed that the deposition effect of droplets between different tree shapes was significant. The flying height of $1.2 \mathrm{~m}$ from the top of the tree was the best operating height, and the droplet deposition density was the highest. Chen et al. ${ }^{[15]}$ used various types of nozzle to investigate the disease and pest control effect of aerial spraying on rice, and proved that selecting a nozzle with a small atomizing droplet size for UAV could improve the control effect of planthoppers. It is obvious that the influencing factors of aerial application are complex and diverse, and there are less basic researches on the atomization mechanism of aerial spraying at present ${ }^{[16-18]}$, especially the accurate research on deposition and drift characteristics of spray droplets. Moreover, rarely scholars pay attention to the determination of the effective swath of droplets $^{[19,20]}$.

The effective swath width of droplets is the basis of aerial spraying research, and its accurate assessment is of great significance to the planning of operation route and the improvement of spray operation quality ${ }^{[21]}$. Chen et al. ${ }^{[22]}$ conducted a study on the evaluation methods of the effective swath width of different UAVs, and the results showed the effective swath width evaluation methods was determined according to the range of the droplet size. Yang et al. ${ }^{[23]}$ analyzed the influence of the UAV downwash airflow on the effective swath width and simulated the test results. Zhang et al. ${ }^{[24]}$ carried out a research on the droplet deposition distribution and drift of M-18B and Thrush $510 \mathrm{G}$ fixed-wing aircraft. The effective swath width of the two aircrafts were accurately measured, and the uniformity of droplet deposition distribution under different operating conditions was clarified. Yao et al. ${ }^{[25,26]}$ conducted a variable spray effect study on the AS350B3e helicopter equipped with the AG-NAV Guía system based on the effective swath width measurement, and the droplet deposition and drift distribution characteristics of the helicopter were measured. The results showed that the flight speed has no significant effect on the uniformity of the droplet deposition distribution in the effective swath area. But when the helicopter was spraying at a flying height of $5 \mathrm{~m}$, a buffer zone of at least $50 \mathrm{~m}$ should be reserved. Due to the limitation of the aircraft type, spray equipment and flight parameters, the results of the above studies are only partly representative and have limited applicability. At the same time, related studies generally lack the analysis of the accumulation of droplets and the spatial vertical drift.

In order to accurately understand the movement characteristics of the droplets in the process of aerial application, this paper took the manned helicopter and its matching fan-shaped spray nozzle as the research object, and carried out a single-pass application test based on the determination of the effective swath width. By setting ground and spatial sampling points, the accurate effective swath width of the helicopter spray operation was obtained, and the cumulative and spatial drift characteristics of the droplet deposition when the helicopter used different aerial nozzles were systematically analyzed. The research results can provide a reference for optimizing the aerial spraying plan and improving the efficiency of spray operations.

\section{Materials and methods}

\subsection{Manned helicopter and carrying equipment}

All tests were made using a Bell206L4 helicopter (Bell
Helicopter Textron Inc., Fort Worth, USA), with the fuselage of $10.56 \mathrm{~m} \times 2.33 \mathrm{~m} \times 3.04 \mathrm{~m}$ (Length $\times$ width $\times$ height $).$ And the main rotor diameter was $11.28 \mathrm{~m}$, tail rotor diameter was $1.65 \mathrm{~m}$, with the rotor speed of $395 \mathrm{r} / \mathrm{min}$. The helicopter was equipped with a Simplex 7900 spray system (Simplex Manufacturing Company Inc., Auburn, USA) for precision spray control. The tank was located under the belly of the machine with the maximum capacity of $600 \mathrm{~L}$, and the spray boom connected with the tank was $8.6 \mathrm{~m}$ long. The tested aerial nozzles were hydraulic $\mathrm{CP}$ flat nozzles (CP Products, Inc., Tempe, AZ, USA) with a spray fan angle of $80^{\circ}$, the spray pressure was $310-345 \mathrm{kPa}$. The nozzles were arranged backwards in the horizontal direction with the number of 51. The helicopter was also equipped with an airborne BeiDou real time kinematic (RTK) differential system with precise differential positioning function, which can record flight parameters in real time and plot the actual operation trajectory as a reference analysis of spraying effects.

\subsection{Spray reagent and sample collection card}

The spray reagent was a $5 \%$ urea aqueous solution with a preparation volume of $400 \mathrm{~L}$. The droplet collection card was water sensitive paper (WSP) (Chongqing Liuliushanxia Plant Protection Technology Co., Ltd, Chongqing, China) with the size of $80 \mathrm{~mm} \times 30 \mathrm{~mm}$.

\subsection{Test site and layout}

The experiment was conducted in Qulai Town $\left(114^{\circ} 55^{\prime} \mathrm{E}\right.$, $27^{\circ} 05^{\prime} \mathrm{N}$ ) of Ji'an City, Jiangxi Province, China, and the spraying target was grassland with a grass height of $5-10 \mathrm{~cm}$. As shown in Figure 1a, there were three $80 \mathrm{~m}$ long droplet sampling lines with a spacing of $40 \mathrm{~m}$ that were perpendicular to the helicopter flight route. Each sampling line was marked in sequence from west to east (W-E) of -40 to $40 \mathrm{~m}, 0 \mathrm{~m}$ was the flight route of helicopter, and the flight route was from north to south (N-S). The area from -20 to $20 \mathrm{~m}$ was the effective swath width test area (Target area). A piece of WSP was arranged at intervals of $1 \mathrm{~m}$ and $30 \mathrm{~cm}$ from the ground. The area of -40 to $-20 \mathrm{~m}$ and 20 to $40 \mathrm{~m}$ were the sampling areas for the spatial drift distribution of droplets (Non-target area). The sampling points were separated by $2 \mathrm{~m}$, each point was arranged vertically with three pieces of WSP, and the height above the ground was $30 \mathrm{~cm}, 100 \mathrm{~cm}$ and $180 \mathrm{~cm}$. All WSPs were arranged face up, and the helicopter spray test site was shown in Figure $1 b$.

Single-pass application $(1 \#-3 \#)$ was carried out three times in a sequence of nozzle $\mathrm{CP} 02, \mathrm{CP} 03$, and $\mathrm{CP} 04$. The flight height and velocity of all three tests were set as $11 \mathrm{~m}$ and $17 \mathrm{~m} / \mathrm{s}$ according to actual operating experience, and the flight operation parameters were recorded in real time by the airborne BeiDou RTK differential system. At the same time, a Kestrel 5500 Link micro weather station (Nielsen-Kellerman Co., Boothwyn, USA) was set up $2 \mathrm{~m}$ from the ground outside the test area for real-time recording of meteorological information such as wind speed, wind direction, atmospheric pressure, temperature and humidity in the natural environment during the test. The test settings and related operation parameters were summarized in Table 1.

\subsection{Data analysis}

After each spray application test, the WSPs were analyzed by image processing software DepositScan (USDA. Wooster, USA). The droplet deposition of each sampling point can be obtained. Meanwhile, mean deposition and coefficient of variation (CV) were also calculated. The CV is used to characterize the uniformity of deposition distribution. The smaller coefficient of variation, the more uniform of droplet deposition distribution is ${ }^{[27,28]}$. 


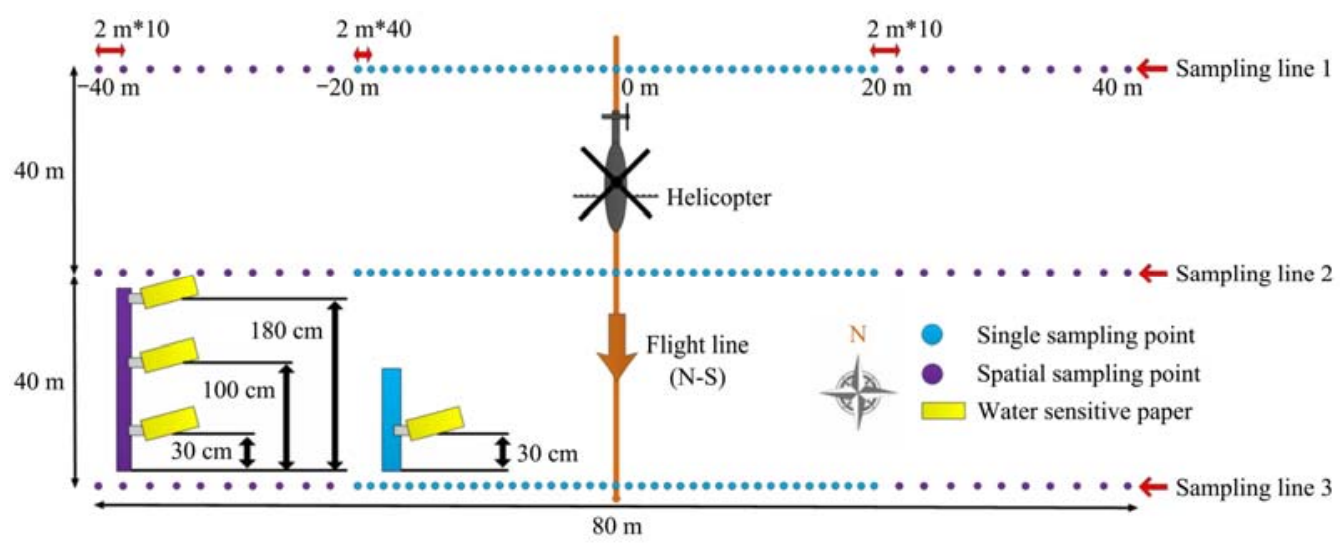

a. Layout of sampling for field test

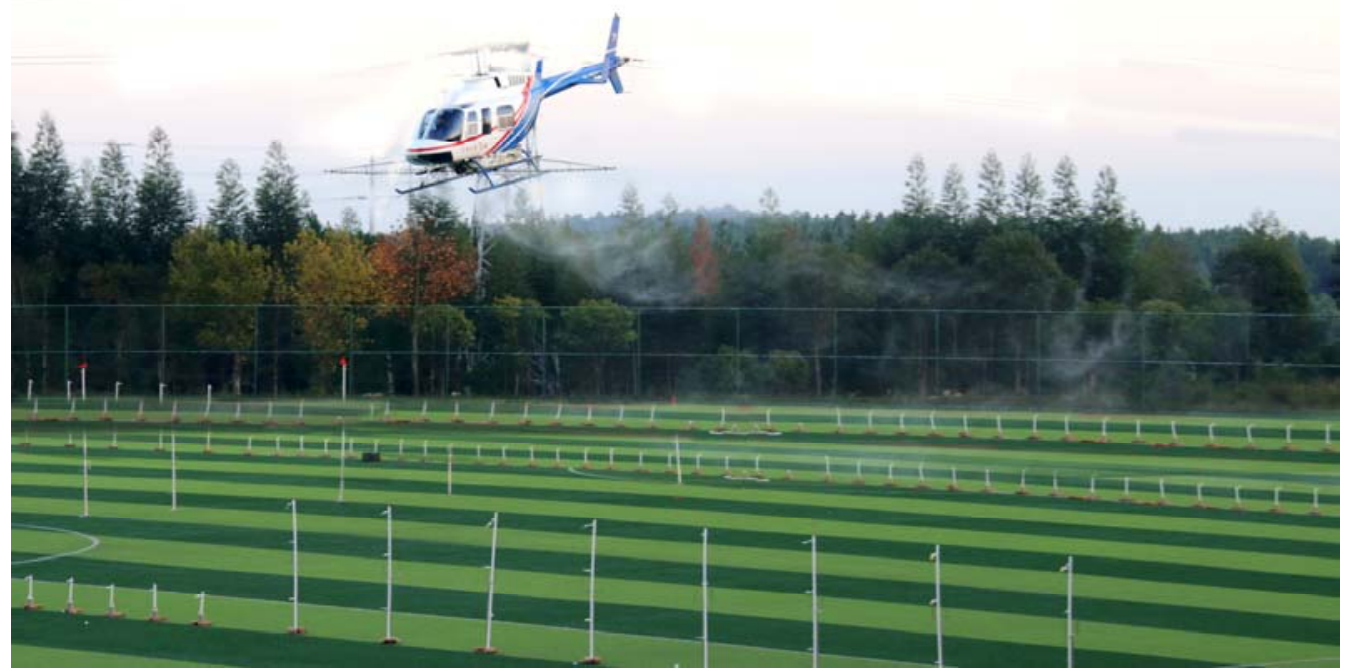

b. Helicopter spray test site

Figure 1 Schematic diagram of test

Table 1 Summary of test operation parameters

\begin{tabular}{ccccccc}
\hline Test & Nozzle & $\begin{array}{c}\text { Spray pressure } \\
/ \mathrm{kPa}\end{array}$ & $\begin{array}{c}\text { Total spray flow rate } \\
/ \mathrm{L} \cdot \mathrm{min}^{-1}\end{array}$ & $\begin{array}{c}\text { Wind velocity and wind } \\
\text { direction } / \mathrm{m} \cdot \mathrm{s}^{-1}\end{array}$ & $\begin{array}{c}\text { Temperature } \\
/{ }^{\circ} \mathrm{C}\end{array}$ & $\begin{array}{c}\text { Flight height } \\
/ \mathrm{m}\end{array}$ \\
\hline $1 \#$ & $\mathrm{CP02}$ & 345 & 51.01 & $0.5 / \mathrm{NE}$ & 17.9 & 12.11 \\
$2 \#$ & $\mathrm{CP03}$ & 330 & 77.01 & $0.9 / \mathrm{N}$ & 17.8 & 10.87 \\
$3 \#$ & $\mathrm{CP04}$ & 310 & 98.43 & $1.2 / \mathrm{NE}$ & 17.58 & 16.4 \\
\hline
\end{tabular}

\subsection{Determination method of effective swath}

To determine the effective swath, the research methods of fixed-wing aircraft ${ }^{[24]}$ and manned helicopter ${ }^{[26]}$ and industrial standard MH/T 1040-2011 (2011) ${ }^{[29]}$ were referred. We took the deposition of $1.000 \mu \mathrm{L} / \mathrm{cm}^{2}$ as a judging standard. The farthest sampling point which deposition volume reached to $1.000 \mu \mathrm{L} / \mathrm{cm}^{2}$ on both sides of the route were defined as the starting and ending point of the effective swath area. The distance between these two points was the effective swath width.

\subsection{Calculation method of deposition cumulated}

In this paper, the distribution of deposition along the sampling distance was drawn by taking the deposition volume as the ordinate and the sampling point as the horizontal coordinate ${ }^{[30]}$. The aircraft route at $0 \mathrm{~m}$ was set as the starting point, and the deposition volume on both sides of the route was accumulated to obtain the deposition accumulation percentage curve (Percentage A). The deposition accumulation corresponding to the sampling points on both sides of the effective swath boundary was called percentage of effective swath boundary in the left area (PEL) and percentage of effective swath boundary in the right area (PER), respectively. In addition, the accumulation percentage curve of the entire sampling line (Percentage B) can also be obtained by sequentially accumulating the deposition from -40 to $40 \mathrm{~m}$. When the cumulative deposition volume reaches $50 \%$ of the total cumulative deposition volume, the corresponding sampling point position was called cumulative midpoint of droplet deposition (CMD).

\section{Results and discussion}

\subsection{Effective swath and corresponding deposition effect}

The effective swath width and droplet deposition results for each sampling line of each test were shown in Table 2 . The results indicated that the average overall deposition volume of each sampling line of the three tests was $0.626-1.448 \mu \mathrm{L} / \mathrm{cm}^{2}$, and the difference between each test was obvious, the main reason was that the difference in the droplet size and flow rate of three types of nozzle. The measured effective swath width of the helicopter was 20 to $32 \mathrm{~m}$, and the start and end range were scattered in the range of -19 to $18 \mathrm{~m}$ of the sampling line. Among them, the effective swath width of test $1 \#$ were $29 \mathrm{~m}(1 \#-1), 27 \mathrm{~m}$ (1\#-2) and $20 \mathrm{~m}$ (1\#-3), respectively, and the average effective swath width was $25.3 \mathrm{~m}$, which was quite different from test $2 \#$ and test $3 \#$. The effective swath width of test $2 \#$ were $32 \mathrm{~m}$ (2\#-1), $29 \mathrm{~m}(2 \#-2)$ and 
$28 \mathrm{~m}(2 \#-3)$, respectively, and the average effective swath width was $29.7 \mathrm{~m}$. The effective swath width of test $3 \#$ were $31 \mathrm{~m}$

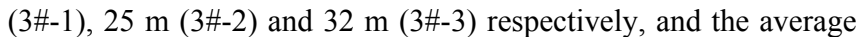
effective swath width was $29.3 \mathrm{~m}$. It can be found that when the helicopter used $\mathrm{CP} 03$ and $\mathrm{CP} 04$ nozzles for spraying operations, the effective swath width was larger and the values were consistent. At the same time, the average deposition in the effective swath area of each sampling line was $1.208-2.735 \mu \mathrm{L} / \mathrm{cm}^{2}$, and the difference between the three tests was also obvious. In addition, the $\mathrm{CV}$ of the deposition in the effective swath area of each sampling line was 40.17-61.05\%, which indicated a better droplet deposition uniformity.

Table 2 Effective swath and corresponding deposition effect of each sampling line

\begin{tabular}{ccccc}
\hline $\begin{array}{c}\text { Sampling } \\
\text { line }\end{array}$ & $\begin{array}{c}\text { Mean deposition } \\
\text { of overall } \\
\text { sampling } \\
\text { line/ } \mu \mathrm{L} \cdot \mathrm{cm}^{-2}\end{array}$ & $\begin{array}{c}\text { Effective } \\
\text { swath width } \\
\text { (start and end } \\
\text { range } / \mathrm{m}\end{array}$ & $\begin{array}{c}\text { Mean deposition } \\
\text { of effective } \\
\text { swath width } \\
\text { area/ } \mu \mathrm{L} \cdot \mathrm{cm}^{-2}\end{array}$ & $\begin{array}{c}\text { CV of deposition } \\
\text { in effective swath } \\
\text { width area/\% }\end{array}$ \\
\hline $1 \#-1$ & 0.731 & $29(-15-14)$ & 1.208 & 43.41 \\
$1 \#-2$ & 0.851 & $27(-19-08)$ & 1.527 & 40.87 \\
$1 \#-3$ & 0.626 & $20(-10-10)$ & 1.375 & 54.99 \\
$2 \#-1$ & 0.906 & $32(-19-13)$ & 1.491 & 59.86 \\
$2 \#-2$ & 0.809 & $29(-12-17)$ & 1.448 & 40.17 \\
$2 \#-3$ & 0.897 & $28(-10-18)$ & 1.581 & 57.66 \\
$3 \#-1$ & 1.448 & $31(-16-15)$ & 2.504 & 53.54 \\
$3 \#-2$ & 1.273 & $25(-15-10)$ & 2.735 & 44.09 \\
$3 \#-3$ & 1.362 & $32(-18-14)$ & 2.330 & 61.05 \\
\hline
\end{tabular}

In order to further characterize the droplet deposition characteristics of each test, the overall deposition data of the effective swath area was analyzed (Table 3). It can be found that the measured standard deviation and $\mathrm{CV}$ of the deposition in the effective swath area were relatively small, indicating that the differences between the sampling lines of the same tests were small. However, the average deposition in the effective swath area of the three tests was quite different, and the average deposition of the three tests were $1.370 \mu \mathrm{L} / \mathrm{cm}^{2}$ (test $1 \#$ ), $1.507 \mu \mathrm{L} / \mathrm{cm}^{2}$ (test $2 \#$ ) and $2.523 \mu \mathrm{L} / \mathrm{cm}^{2}$ (test $3 \#$ ), respectively. The increasing trend was also consistent with the nozzle orifice sizes from small to large.

Table 3 The overall deposition results in effective swath area for each test

\begin{tabular}{lccc}
\hline Measured value & Test 1\# & Test 2\# & Test 3\# \\
\hline Mean deposition $/ \mu \mathrm{L} \cdot \mathrm{cm}^{-2}$ & 1.370 & 1.507 & 2.523 \\
Standard deviation of deposition & 0.16 & 0.07 & 0.20 \\
CV of deposition $\%$ & 11.65 & 4.50 & 8.06 \\
\hline
\end{tabular}

\subsection{Distribution and cumulative effect of droplet deposition}

Based on the determination of the effective swath width, the distribution and cumulative of droplet deposition at the sampling points of each sampling line were further analyzed (Figure 2). The maximum droplet deposition volume of the sampling points of the three tests were $3.519 \mu \mathrm{L} / \mathrm{cm}^{2}(1 \#-3,-8 \mathrm{~m}), 3.717 \mu \mathrm{L} / \mathrm{cm}^{2}$ (2\#-1, $-3 \mathrm{~m})$ and $4.921 \mu \mathrm{L} / \mathrm{cm}^{2}(3 \#-1,6 \mathrm{~m})$, respectively. For the test $1 \#$, $2 \#$ and $3 \#$, the maximum droplet deposition volume of the sampling points showed a gradual increase trend. For the sampling line, the droplet deposition distribution of each sampling line showed a trend of gradually decreasing from the position of the flight route $(0 \mathrm{~m})$ to the positions on both sides of the route $(-40 \mathrm{~m}$, $40 \mathrm{~m})$. It can also be clearly seen from Figure 2 that since most of the droplets were concentrated near the route and the adjacent locations of the effective swath area, both PEL and PER were at a level of more than $53 \%$, which indicated that the droplets were main deposited in the effective swath area, and the actual proportion of the droplet deposition in this area had reached $53 \%$ or even higher of the total droplet deposition. Further, the PEL of test $1 \#$ was in the range of $66.27 \%-85.91 \%$, the PER was in the range of $53.66 \%-82.61 \%$; the PEL of test $2 \#$ was in the range of $75.84 \%-81.48 \%$, and the PER was in the range of $75.71 \%-82.53 \%$; the PEL of test $3 \#$ was in the range of $73.40 \%-85.43 \%$, and the PER was in the range of $89.37 \%-93.40 \%$. Especially, the PER of test $3 \#$ even reached over $89 \%$, indicating that only less than $11 \%$ of the droplets in the right area of the route drifted.

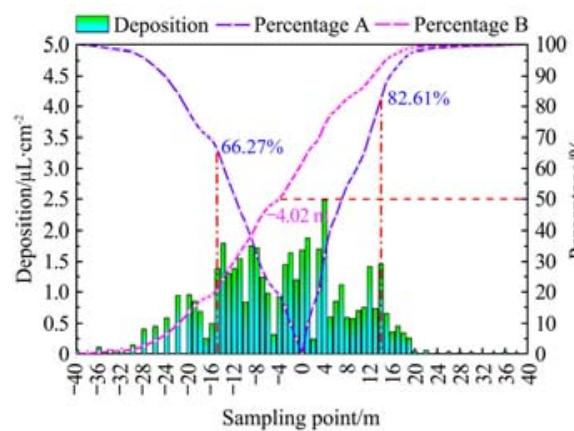

a. $1 \#-1$

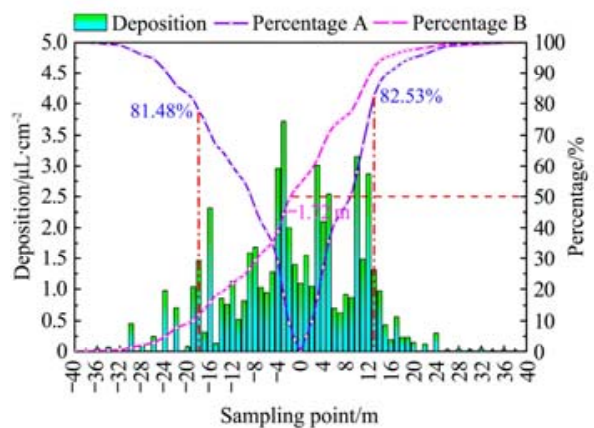

d. $2 \#-1$

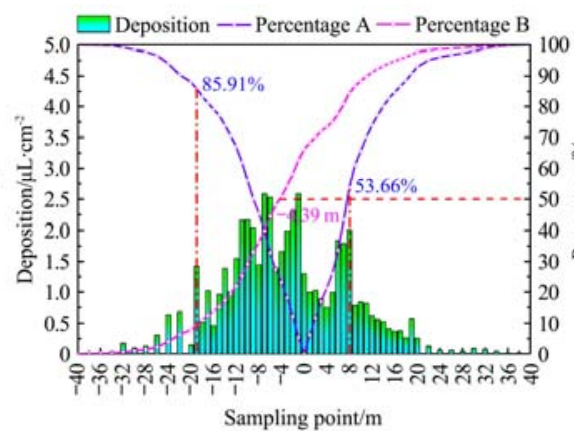

b. $1 \#-2$

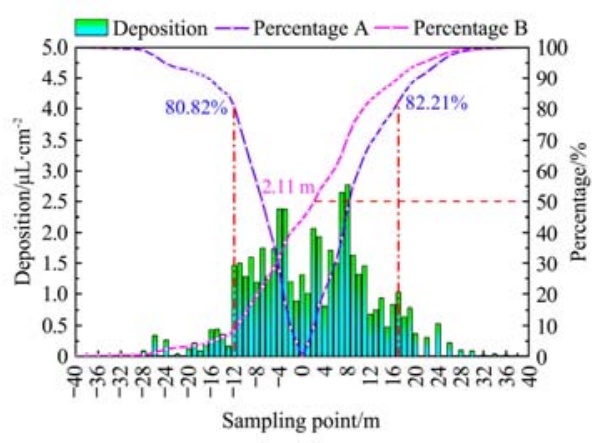

e. $2 \#-2$

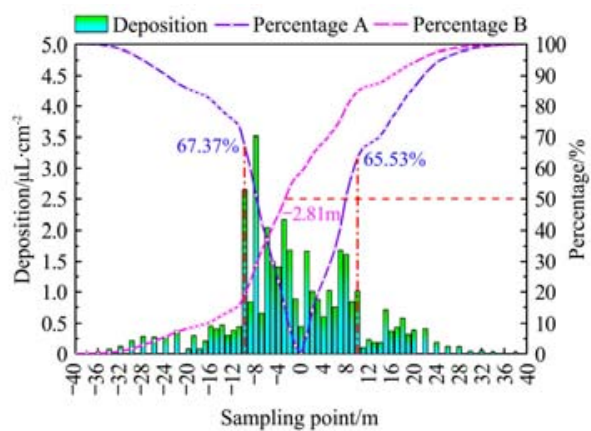

c. $1 \#-3$

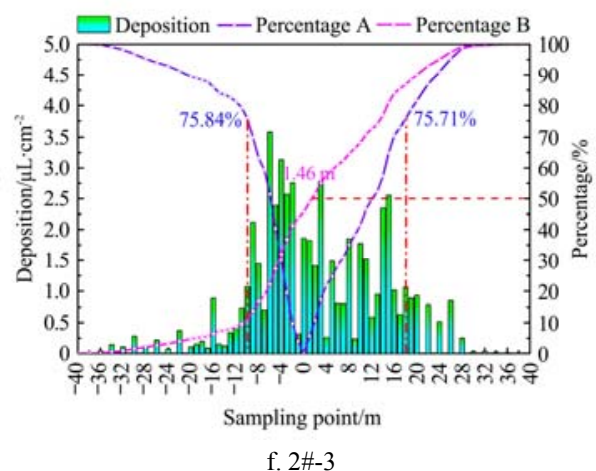



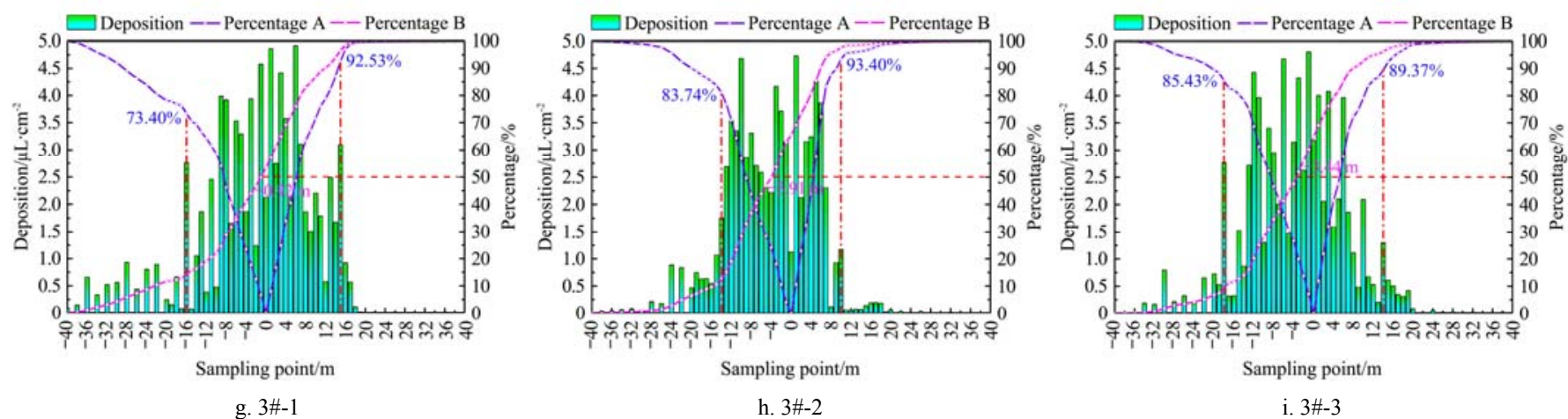

Figure 2 Distribution and cumulative effect of droplet deposition in each sampling line

In addition, the study also found that the CMD of each sampling line was not at $0 \mathrm{~m}$ on the route. The CMD of each sampling line of test $1 \#$ and test $3 \#$ were in the left area of the route, and the offset distance of $1 \#-2$ was the largest $(4.39 \mathrm{~m})$. For test $2 \#$, the CMD of 2\#-1 was also in the left area of the route, but the CMDs of 2\#-2 and 2\#-3 were in the right area of the route with the offset distances of $2.11 \mathrm{~m}$ and $1.46 \mathrm{~m}$, respectively. The possible reasons for the result were analyzed from the perspective of airflow. The direction of the flight route was from north to south, and the natural wind was northeast wind during test $1 \#$ and test $3 \#$ operations. Affected by the wind direction, more droplets were deposited on the left area of the route (downwind area). During the test 2\# operation, the natural wind was north wind with the direction paralleled to the route. At this time, the droplet settlement was mainly affected by the airflow of the helicopter rotor. The droplets were deposited randomly on both sides of the route, and CMD was also randomly scattered on both sides of the route.

\subsection{Analysis of deposition percentage}

According to the spray effect, the effective swath area \& drift area and the route left area $\&$ route right area were divided on each sampling line. The effective depositions and the proportions of drift (invalid deposition) in each corresponding area were counted. Figure 3 showed the distribution of deposition in each area.

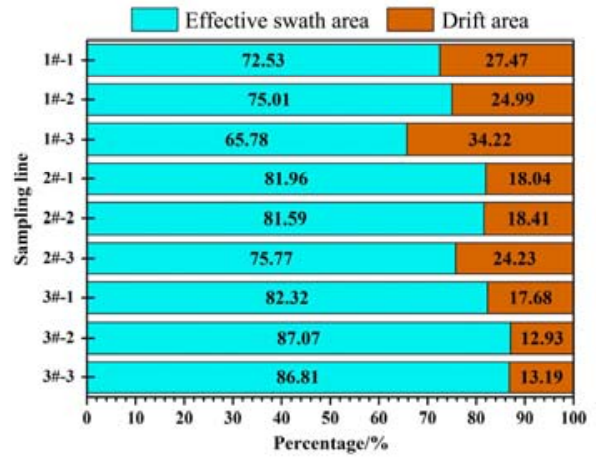

a. Percentage of total droplet deposition

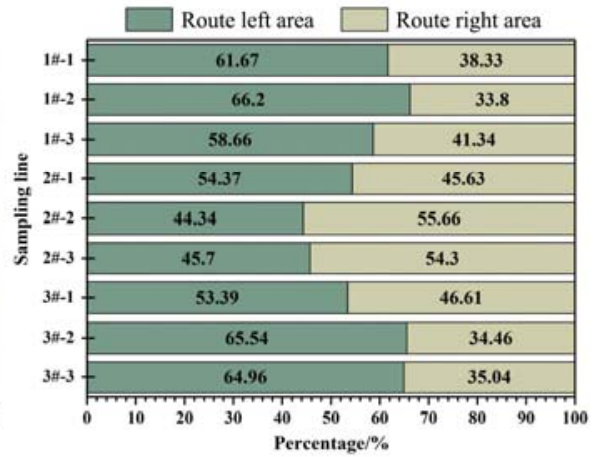

b. Percentage of single-sided droplet deposition

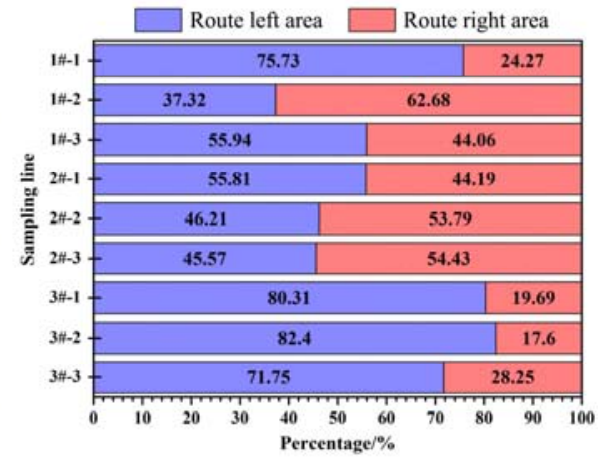

c. Percentage of single-sided drift

Figure 3 The distribution percentage of the deposition in each area

From Figure 3a, the deposition percentage of the effective swath area of test $1 \#$ was in the range of $65.78 \%-75.01 \%$; the deposition percentage of the effective swath area of test $2 \#$ was in the range of $75.77 \%-81.96 \%$; and the deposition percentage of the effective swath area of test $3 \#$ was in the range of $82.32 \%-87.07 \%$. It can be clearly found that the percentage of droplet deposition in the effective swath area increased with the the nozzle orifice size, which also indicated that the droplets of small size were more likely to drift. In addition, the study also found that the percentage of droplet deposition (Figure $3 \mathrm{~b}$ ) and drift percentage (Figure 3c) on the left area of the flight route of test $1 \#$ and test $3 \#$ were significantly higher than those on the right area of the flight route, but the test $2 \#$ reflected a certain random volatility. This was consistent with the previously results of CMD, which was also caused by the influence of natural wind and aircraft trajectory. At the same time, the above-mentioned results of droplet deposition accumulation analysis can be further explained.

\subsection{Distribution of spatial droplet drift}

In this test, areas of -40 to $-22 \mathrm{~m}$ and 22 to $40 \mathrm{~m}$ were the sampling areas for the spatial drift distribution of droplets. For single-pass application, the droplets deposited in this area were invalid. If the deposition volume was too large in this area, it was easy to form phytotoxicity, and lead to low effective utilization of pesticides, then affected the actual efficacy. Figure 4 presented the deposition distribution characteristics of the spatial sampling points arranged in the non-target areas on both sides of the sampling line. It can be seen from Figure 4 that the average droplet drift of each layer by test $1 \#$ was between 0.050 $0.283 \mu \mathrm{L} / \mathrm{cm}^{2}$. And the droplet drifts of $1 \#-1$ and $1 \#-2$ measured in the upper, middle and lower layers of the -40 to $-22 \mathrm{~m}$ area were significantly higher than those measured at the corresponding position in the 22 to $40 \mathrm{~m}$ area. However, the difference of droplet drift between the above two areas of 1\#-3 was small. The average droplet drift of each layer by test $2 \#$ was between 0.026 $0.264 \mu \mathrm{L} / \mathrm{cm}^{2}$. And the droplet drifts of $2 \#-1$ measured in the upper, middle and lower layers of the -40 to $-22 \mathrm{~m}$ area were significantly higher than those measured at the corresponding position in the 22 to $40 \mathrm{~m}$ area, and the droplet drift of $2 \#-2$ and 2\#-3 in the two regions showed opposite trends. The average droplet drift of each layer by test $3 \#$ was between 0.007 $0.538 \mu \mathrm{L} / \mathrm{cm}^{2}$. In addition, the droplet drift measured in the upper, middle and lower layers of all the sampling lines of test $3 \#$ in the 
-40 to $-22 \mathrm{~m}$ area were significantly higher than those measured in the corresponding position in the 22 to $40 \mathrm{~m}$ area. At the same time, the volume of droplet drift in the middle layer was generally higher than other layers.
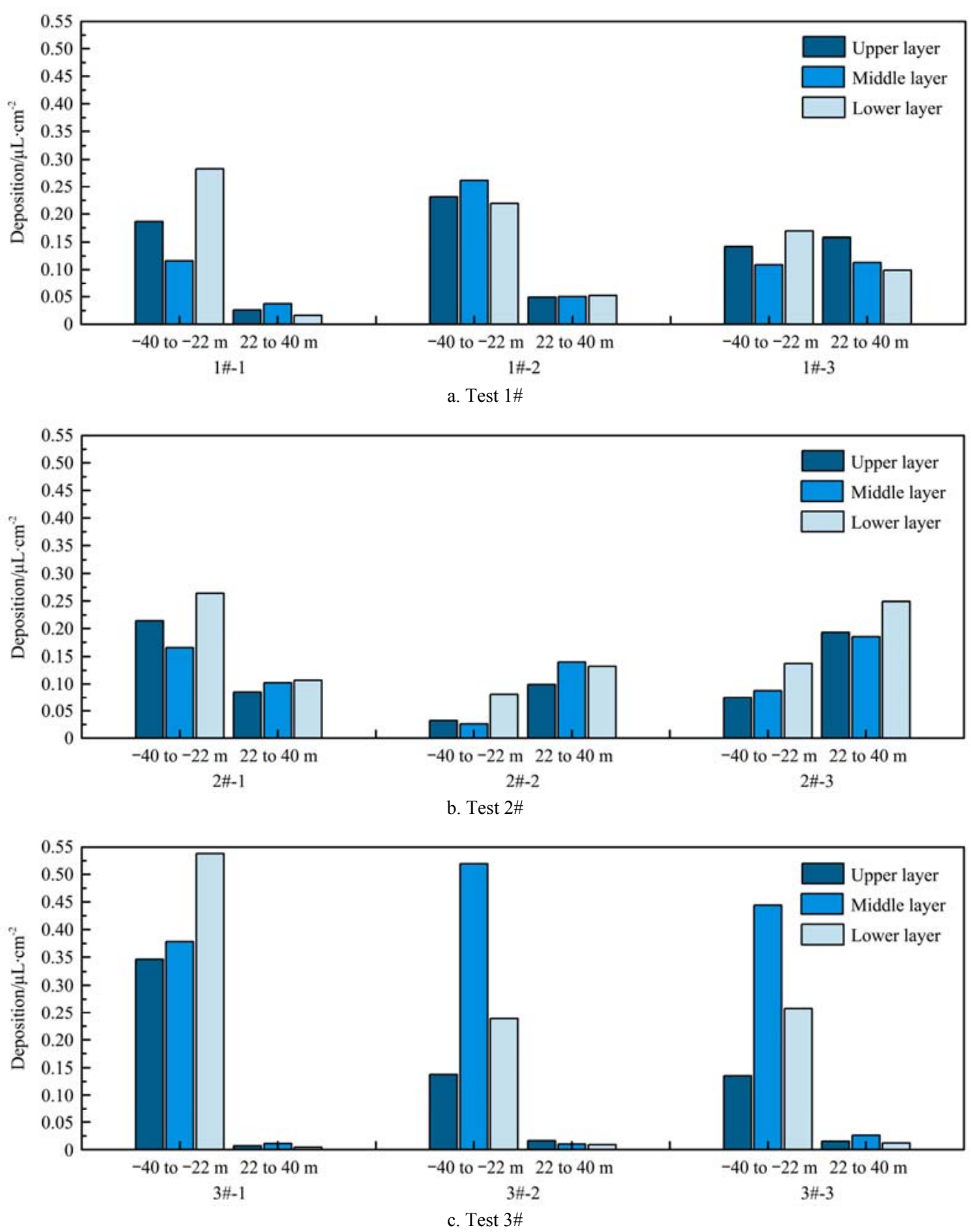

Figure 4 Mean value of each layer droplet deposition in the spatial sampling area on both sides

Figure 5 showed the overall spatial variation trend and distribution percentage of the droplet drift volume of each test. As shown in Figure 5a, from the sampling position of upper-layer to the lower-layer, the drift volume of test $1 \#$ showed a trend of decreasing and then increasing; the drift volume of test 2\# showed a gradual increase trend; the drift volume of test $3 \#$ showed a trend of increasing and then decreasing. At the same time, the average drift of the upper layer by test 1 \# was the highest $\left(0.132 \mu \mathrm{L} / \mathrm{cm}^{2}\right)$, which indicated that the droplets with smaller droplet size were more likely to gather in the space above the non-target area. The average drift of the test $3 \#$ in the middle layer was $0.231 \mu \mathrm{L} / \mathrm{cm}^{2}$, which was the highest value of all the spatial sampling positions of each test, and it was also in line with the above-mentioned research results of the middle layer droplet drift of test 3\#. As shown in Figure 5b, the drift proportions of the sampling positions in each layer of test $1 \#$ were relatively evenly distributed, with a range of only $6.52 \%$; test $2 \#$ had the highest drift at lower layer of the sampling position $(40.86 \%)$; test $3 \#$ had the highest drift at middle layer of the sampling position $(44.7 \%)$. It can also be concluded that the droplets sprayed by $\mathrm{CP} 04$ nozzles were easy to gather and drift in the middle layer of the non-target area due to their large droplet size. Most of the droplets from CP03 nozzles was settled down at the lower layer of the non-target area, and the droplets sprayed by $\mathrm{CP} 02$ nozzles were more evenly distributed in the space of non-target area due to their smaller droplet size.

\section{Conclusions}

In this paper, the character of droplet deposition and drift by Bell206L4 helicopter with single-pass application had been systematically studied. The aim was to determine the effective swath width and deposition distribution of the helicopter when using different nozzles, as well as the spatial drift of the droplets in the non-target area. The conclusions were as follows: 


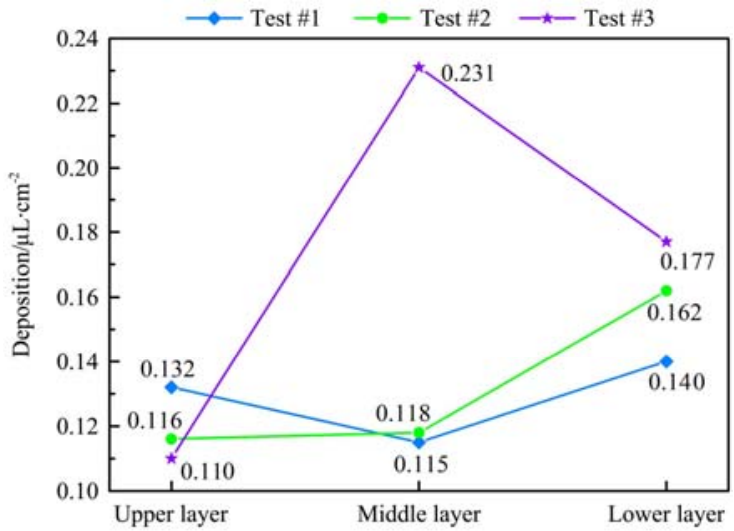

a. Spatial variation trend of droplet drift

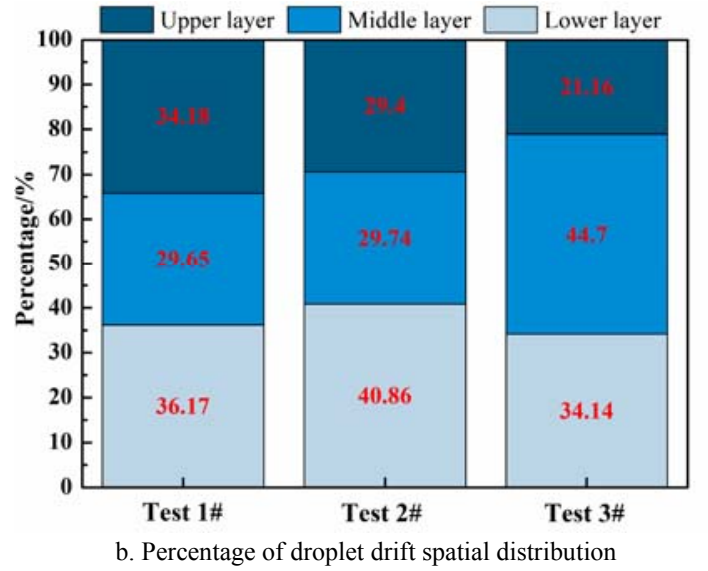

Figure 5 Spatial variation trend and distribution percentage of the droplet drift volume for each test

1) Under the conditions of helicopter flight operations with an average flight altitude of $11 \mathrm{~m}$ and an average flight speed of $17 \mathrm{~m} / \mathrm{s}$, the average effective swath width of test 1 \# (CP02) was $25.3 \mathrm{~m}$; the average effective swath width of test 2 \# (CP03) was $29.7 \mathrm{~m}$; the average effective swath width of test 3 \# (CP04) was $29.3 \mathrm{~m}$.

2) The average deposition in the effective swath area of each sampling line was $1.208-2.735 \mu \mathrm{L} / \mathrm{cm}^{2}$. The average depositions in the effective swath area of the three tests were $1.370 \mu \mathrm{L} / \mathrm{cm}^{2}$ (test $1 \#$ ), $1.507 \mu \mathrm{L} / \mathrm{cm}^{2}$ (test $2 \#$ ) and $2.523 \mu \mathrm{L} / \mathrm{cm}^{2}$ (test 3\#), respectively, and the increasing trend was also consistent with the test setting sequence of the nozzle orifice sizes from small to large.

3) Most of the droplets in each spray operation were concentrated near the route and the adjacent locations of the effective swath area, and the actual proportion of the droplet deposition in this area had reached $53 \%$ of the total droplet deposition or even higher. In addition, affected by natural wind, the CMD of each sampling line was not at $0 \mathrm{~m}$ on the route, and would deviate to a certain extent to the downward wind direction.

4) For spray droplets with different droplet sizes, the droplets of small size were more likely to drift, and the increase of orifice size by nozzle can help reduce drift. The study also found that the percentage of droplet deposition in the effective swath area increased with the nozzle orifice size, especially the percentage of droplet deposition in the effective swath area by test $3 \#$ had reached more than $82 \%$.

5) Small-sized droplets usually gathered in the upper space of the non-target area, larger-sized droplets were easy to gather in the middle space of the non-target area, the medium-sized droplets were trend to gather in the lower space of the non-target area.

\section{Acknowledgments}

We deeply thank for the Liaoning Province Science and Technology Plan Project (2019JH2/10200002), National Key Technologies Research and Development Program (2016YFD0200700), 111 Project (D18019), Key R\&D projects in Hainan Province (ZDYF2020195). Thanks to Jiangxi Tianren Ecology Co., Ltd for strong support.

\section{[References]}

[1] Godfray H C, Beddington J R, Crute I R, et al. Food security: the challenge of feeding 9 billion people. Science, 2010; 327(5967): 812-818. doi: $10.1126 /$ science.1185383.

[2] Del Cerro J, Cruz Ulloa C, Barrientos A, et al. Unmanned aerial vehicles in agriculture: A survey. Agronomy, 2021; 11(2): 203. doi: 10.3390/ agronomy11020203.

[3] Lan Y B, Chen S D, Fritz B K. Current status and future trends of precision agricultural aviation technologies. International Journal of Agricultural \& Biological Engineering, 2017; 10(3): 1-17. doi: 10.3965/ j.ijabe.20171003.3088.

[4] Dong Y J, Zeng F W, Yuan J, Zhang G B, Chen Y X, Liu X J, et al. Integrated rice management simultaneously improves rice yield and nitrogen use efficiency in various paddy fields. Pedosphere, 2020; 30(6): 863-73. doi: 10.1016/s1002-0160(20)60042-x.

[5] Dorr G, Hanan J, Adkins S, et al. Spray deposition on plant surfaces: a modelling approach. Functional Plant Biology, 2008; 35(2): 988-996. doi: 10.1071/FP08056.

[6] Hilz E, Vermeer A W P. Spray drift review: The extent to which a formulation can contribute to spray drift reduction. Crop Protection, 2013; 44(1): 75-83. doi: 10.1016/j.cropro.2012.10.020.

[7] Guo S, Li J Y, Yao W X, et al. Distribution characteristics on droplet deposition of wind field vortex formed by multirotor UAV. PLoS ONE, 2019; 14(7), e0220024. doi: 10.1371/journal.pone.0220024.

[8] Thomson S J, Smith L A, Hanks J E. Evaluation of application accuracy and performance of a hydraulically operated variable-rate aerial application system. Transactions of the ASABE, 2009; 52(3): 715-722. doi: 10.13031/2013.27389.

[9] Fritz B K. Meteorological effects on deposition and drift of aerially applied sprays. Transactions of the ASABE, 2006; 49(5): 1295-1301. doi: 10.13031/2013.22038.

[10] Yao W X, Lan Y B, Hoffmann W C, et al. Atomization characteristics of multi-type aerial nozzles in wind tunnel and low airflow velocity condition in manned agricultural helicopter. International Journal of Precision Agricultural Aviation, 2019; 2(1): 9-17. doi: 10.33440/ j.ijpaa.20190201.0031.

[11] Yao W X, Lan Y B, Hoffmann W C, et al. Droplet size distribution characteristics of aerial nozzles by Bell206L4 helicopter under medium and low airflow velocity wind tunnel condition and field verification test. Applied sciences, 2020; 10(6): 2179. doi: 10.3390/app10062179.

[12] Meng Y H, Su J Y, Song J L, et al. Experimental evaluation of UAV spraying for peach trees of different shapes: Effects of operational parameters on droplet distribution. Computers and Electronics in Agriculture, 2020; 170, 105282. doi: 10.1016/j.compag.2020.105282.

[13] Wang J, Lan Y B, Yao W X, et al. Aerial spraying application of multi-rotor unmanned aerial vehicle on areca trees. International Journal of Precision Agricultural Aviation, 2020; 3(4): 51-64. doi: 10.33440/ j.ijpaa.20200304.134.

[14] Tang Y, Hou C J, Luo S M, et al. Effects of operation height and tree shape on droplet deposition in citrus trees using an unmanned aerial vehicle. Computers \& Electronics in Agriculture, 2018; 148:1-7. doi: 10.1016/ j.compag.2018.02.026.

[15] Chen P C, Lan Y B, Huang X Y, et al. Droplet deposition and control of planthoppers of different nozzles in two-stage rice with a quadrotor unmanned aerial vehicle. Agronomy, 2020; 10(2): 303. doi: 10.3390/ agronomy10020303.

[16] Rahman M F F, Fan S R, Zhang Y, et al. A comparative study on application of unmanned aerial vehicle systems in agriculture. Agriculture, 2021; 11(1): 22. doi: 10.3390/agriculture11010022.

[17] Xu T Y, Yu F H, Cao Y L, et al. Vertical distribution of spray droplet deposition of plant protection multi rotor UAV for japonica rice. Transactions of the Chinese Society for Agricultural Machinery, 2017; 48(10): 101-107. doi: 10. 6041/j.issn. 1000-1298. 2017.10.012. (in Chinese) 
[18] Zeng A J, Wang C L, Song J L, et al. Effects of nozzle types, adjuvants and environmental conditions on spray drift potential of unmanned aerial vehicles in a wind tunnel. Chinese Journal of Pesticide Science, 2020; 22(2): 315-323. doi: 10.16801/j.issn.1008-7303.2020.0067. (in Chinese)

[19] Yu F H, Feng S, Yao W X, et al. BAS-ELM based UAV hyperspectral remote sensing inversion modeling of rice canopy nitrogen content. International Journal of Precision Agricultural Aviation, 2020; 3(3): 59-64. doi: 10.33440/j.ijpaa.20200303.105.

[20] Zhang R R, Li Y, Yi T C, Chen L P. Design and experiments of control system of variable pesticide application for manned helicopter. Journal of Agricultural Mechanization Research, 2017; 10: 124-127. doi: 10.13427/j.cnki.njyi.2017.10.025. (in Chinese)

[21] Chen S D, Lan Y B, Li J Y, et al. Effect of spray parameters of small unmanned helicopter on distribution regularity of droplet deposition in hybrid rice canopy. Transactions of the Chinese Society of Agricultural Engineering, 2016; 32(17): 40-46. doi: 10.11975/ j.issn.1002-6819.2016.17.006. (in Chinese)

[22] Chen S D, Lan Y B, Li J Y, et al. Evaluation and test of effective spraying width of aerial spraying on plant protection UAV. Transactions of the Chinese Society of Agricultural Engineering, 2017; 33(7): 82-90. doi: 10.11975/j.issn.1002-6819.2017.07.011. (in Chinese)

[23] Yang Z L, Ge L Z, Qi L J, et al. Influence of UAV rotor down-wash airflow on spray width. Transactions of the Chinese Society for Agricultural Machinery, 2018; 2018; 49(1): 116-122. doi: 10.6041/ j.issn.1000-1298.2018.01.014. (in Chinese)

[24] Zhang D Y, Chen L P, Zhang R R, et al. Evaluating effective swath width and droplet distribution of aerial spraying systems on M-18B and Thrush 510G airplanes. International Journal of Agricultural \& Biological Engineering, 2015; 8(2): 21-30. doi:10.3965/j.ijabe.20150802.1493.

[25] Yao W X, Lan Y B, Wang J, et al. Droplet drift characteristics of aerial spraying of AS350B3e helicopter. Transactions of the Chinese Society of Agricultural Engineering, 2017; 33(22): 75-83. doi: 10.11975/ j.issn.1002-6819.2017.22.010. (in Chinese)

[26] Yao W X, Lan Y B, Wen S, et al. Evaluation of droplet deposition and effect of variable-rate application by a manned helicopter with AG-NAV Guía system. International Journal of Agricultural \& Biological Engineering, 2019; 12(1): 172-178. doi: 10.25165/j.ijabe.20191201.4039.

[27] Hoffmann W C. Comparison of three imaging systems for water-sensitive papers. Applied Engineering in Agriculture, 2005; 21(6): 961-964. doi: 10.13031/2013.20026

[28] Sudheer K P, Panda R K. Digital image processing for determining drop sizes from irrigation spray nozzles. Agricultural Water Management, 2000; 45(2): 159-167. doi: 10.1016/S0378-3774(99)00079-7.

[29] MH/T Standards. 1040-2011: Determining application rates and distribution patterns from aerial application equipment, Beijing: MHT, 2011. (in Chinese)

[30] MH/T Standards. 1050-2012: Field measurement methods for Aircraft spray drift, Beijing: MHT, 2012. (in Chinese) 